\title{
The Neutron Time-of-Flight Cross Section Program at the University of Kentucky - Adventures in Analysis II
}

\author{
J.R. Vanhoy ${ }^{1, a}$, S.F. Hicks ${ }^{2, b}$, B.C. Combs $^{2}$, B.P. Crider $^{4}$, A.J. French², E.A. Garza1 ${ }^{1}$, S.L. Henderson², T.J. Howard², \\ S.H. Liu $^{3}$, S. Nigam ${ }^{3}$, R.L. Pecha ${ }^{2}$, E.E. Peters ${ }^{3,4}$, F.M. Prados-Estévez ${ }^{3,4}$, M.T. McEllistrem ${ }^{3,4, c}$, B.J. Rice ${ }^{1}$, T.J. \\ Ross $^{3,4}$, Z.C. Santonil ${ }^{2}$, L.C. Sidwell ${ }^{2}$, J.L. Steves ${ }^{1}$, and S.W. Yates ${ }^{3,4, d}$ \\ ${ }^{1}$ U.S. Naval Academy, Department of Physics, Annapolis, Maryland 21402 USA \\ ${ }^{2}$ University of Dallas, Department of Physics, Irving, Texas 75062 USA \\ ${ }^{3}$ University of Kentucky, Department of Chemistry, Lexington, Kentucky 40506 USA \\ ${ }^{4}$ University of Kentucky, Department of Physics \& Astronomy, Lexington, Kentucky 40506 USA
}

\begin{abstract}
Elastic and inelastic neutron differential cross sections are measured at the University of Kentucky Accelerator Laboratory (www.pa.uky.edu/accelerator/) at incident energies in the fast neutron region. The laboratorys facilities and instrumentation will be described and our measurement and analysis procedures outlined. Many corrections are required for neutron scattering experiments and the analysis utilizes information from many other cross section data sets and model calculations. Exploring and understanding the limitations of the foundational information and procedures are important for controlling the accuracy of the cross section results. We are examining the limitations in neutron detection efficiency, the normalization of $\left(n, n^{\prime} \gamma\right)$ cross sections, background reduction, spectrum stripping techniques, and attenuation and multiple scattering corrections. The resulting differential cross sections provide information on the compound elastic and coupled channels reaction mechanisms important for advanced reactor designs.
\end{abstract}

\section{Introduction}

The USDOE Advanced Fuels program funds R\&D of innovative next generation LWR and future fast systems. High-quality neutron scattering data guide sensitivity analyses of fuel behavior during irradiations, and the long-term performance of surrounding coolants and structural materials. Recent evaluations revealed there are significant disagreements in cross section between the nuclear libraries, especially above $1 \mathrm{MeV}$ where there is a critical lack of measurements.

\section{The Accelerator Facility}

The High Voltage Engineering Corporation single-ended Model 7MV CN Van de Graaff accelerator was purchased with funding from the Commonwealth of Kentucky and the Kentucky Research Foundation and installed at the University of Kentucky in 1963. From the beginning the facility has served as a factory for pulsed monoenergetic fast neutrons. The terminal ion pulsing system can deliver several microamperes of protons, deuterons, ${ }^{3} \mathrm{He}$, or ${ }^{4} \mathrm{He}$ ions on target with $\sim 1 \mathrm{~ns}$ bunch widths. The facility achieves an almost unmatched combination of intense,

\footnotetext{
a e-mail: vanhoy@usna.edu

be-mail: hicks@udallas.edu

ce-mail: marcus@uky.edu

de-mail: yates@uky.edu
}

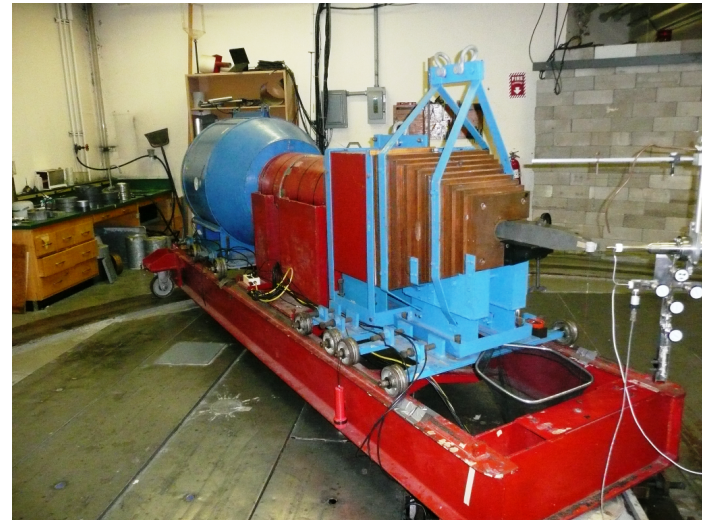

Figure 1. Neutron TOF endstation. The movable carriage contains extensive collimation so the detector only sees neutrons scattered from the sample. The MAIN detector resides in the blue shield which is filled with Li-loaded paraffin and contains $\mathrm{Cd}$ and $\mathrm{Pb}$ rings to reduce thermal neutrons and photons in the vicinity of the detector.

high-energy resolved neutron fluences for basic nuclear structure studies and studies for applications. The laboratory is designed for the fast neutron region a region that is important for both pure and applied nuclear physics.

One experimental hall and a beam line were specially constructed with a 4-meter flight path for neutron time-of- 
flight (TOF) measurements. This room has a high ceiling and a false floor, which covers a 2.8-m deep pit. Quasimonoenergetic fluences of fast neutrons are produced with the ${ }^{3} \mathrm{H}(\mathrm{p}, \mathrm{n}),{ }^{2} \mathrm{H}(\mathrm{d}, \mathrm{n})$, or ${ }^{3} \mathrm{H}(\mathrm{d}, \mathrm{n})$ reactions in a gas cell or the ${ }^{7} \mathrm{Li}(\mathrm{p}, \mathrm{n})$ reaction with an evaporated target. Tritium and deuterium gases are contained in cells ( $\sim 1 \mathrm{~atm})$ constructed of stainless steel and lined with a tantalum foil and beam stopper.

Scattering samples are hung in the neutron fluence about $5.5-7 \mathrm{~cm}$ from the center of the production target and are usually right-circular cylinders containing 0.1 0.5 mole of the enriched isotope of interest. One or more polyethylene samples of similar geometry are used for absolute normalization in the case of $(n, n)$ measurements. Detectors are mounted on a carriage which can be rotated to cover scattering angles up to $155^{\circ}$. The carriage supports a full-length collimation system (Fig. 1).

Neutrons scattered from the sample are detected with a $\mathrm{C}_{6} \mathrm{D}_{6}$ scintillation detector using neutron time-of-flight techniques. Flight paths between 2 and $4 \mathrm{~m}$ are used in most measurements, and pulse-shape discrimination is used to eliminate $\gamma$-ray events in the detector.

De-excitation $\gamma$ rays are detected using a HPGe detector that is surrounded by a BGO Compton-suppression annulus. The detector is mounted on the same rotatable carriage used for neutron detection, although the flight path used for $\gamma$-ray detection is typically about $1.2 \mathrm{~m}$. Unwanted neutron events in the HPGe detector are rejected using TOF techniques. Gamma-ray angular distributions are typically performed from $30^{\circ}$ to $150^{\circ}$ and $\gamma$-ray excitation function measurements are performed at $125^{\circ}$.

Examples of results from the laboratory may be found in other presentations and papers from the CGS-15 conference, in particular, ${ }^{54,56} \mathrm{Fe}$ results are given in Ref. [1].

\section{Measurements and their Limitations}

The University of Kentucky Accelerator Laboratory has 50 years of experience in measuring cross sections. For much of that period, measurements to $10-30 \%$ absolute uncertainty were sufficient for the purposes of the investigations. With the continued interest on safety and advanced reactor designs, these measurements and analysis must be pushed to higher accuracy. Table 1 lists many typical uncertainty contributions during a recent measurement program.

Overall uncertainties achieved during measurements on ${ }^{23} \mathrm{Na}$ were $\sim 8-10 \%$ for elastics and $\sim 13-18 \%$ for inelastics. To achieve the most reliable differential cross sections, it is important to continually examine the effects which generate these uncertainties. In this paper we examine the limitations on extracting yields from TOF spectra.

\section{TOF Spectra on Light Nuclei}

Figure 2 shows TOF spectra for neutrons scattered from ${ }^{12} \mathrm{C}$ at several angles. The dominant peak is rather easy to fit with a gaussian + tail peak shape, however there is a curious feature to the left of the peak. The tendency is to dismiss this feature as some sort of background. How
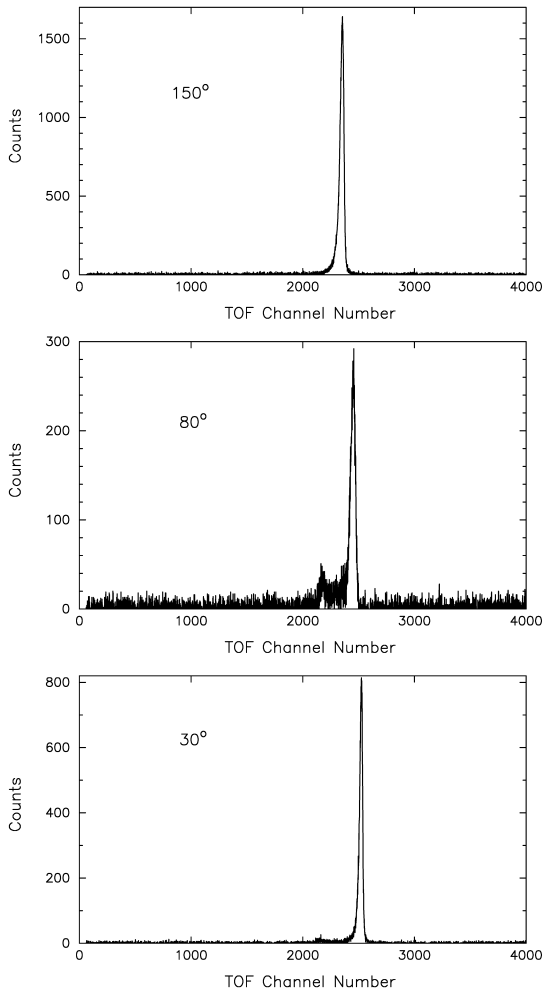

Figure 2. Measured TOF spectra of ${ }^{12} \mathrm{C}(\mathrm{n}, \mathrm{n})$ at $\mathrm{E}_{n}=3.8 \mathrm{MeV}$ at angles of $30^{\circ}$ (bottom), $80^{\circ}$ (middle), and $150^{\circ}$ (top).

one handles this feature can effect the yields in the spectra. Indeed, in earlier 1960s and 1970s spectra, the feature may not have been noticable above backgrounds at the time.

In an effort to understand backgrounds, we began simulations of the neutron production, scattering, and shielding using the code MCNP [2]. Predicted energy spectra for 4.0 MeV neutrons scattering in the ${ }^{12} \mathrm{C}$ sample are shown in Fig. 3.

The main peak is due to a single-scattering event in the sample, as expected. The yield in this peak is reduced from the proper yield because the neutron flux is attenuated entering and leaving the sample. One discovers the extended left-side feature is due to multiple scattering in the sample. Multiple scattering can add to the main peak and alter the tailing (as for $150^{\circ}$ ), or it can create a separate feature to the left (as for $80^{\circ}$ ), or it can produce a wide nearlyinvisible shelf to the left (as for $30^{\circ}$ ). It is interesting that the shape of the double-scattering effect is reminiscent of the shape of the elastic differential cross section in the case of carbon.

Computer codes correct raw measured differential cross sections for the attenuation and multple scattering effects. A proper result requires the input cross sections (yields) include the impact of the attenuation and multiple scattering that is being corrected for in the code. Incorrect reaction yields produce incorrect final cross sections and the problem is angle dependent. To estimate how much 
Table 1. Typical uncertainties in recent ${ }^{23} \mathrm{Na}(\mathrm{n}, \mathrm{n})$ Measurements.

\begin{tabular}{ll}
\hline Issue & Impact on Results \\
\hline Counting Statistics & $<1 \%$ \\
Ability to Extract Yield from TOF Spectrum (elas) & $1-2 \%$ \\
Ability to Extract Yield from TOF Spectrum (inel) & $<10 \%$ \\
Monitoring Neutron Production & $<1 \%$ \\
Sample Mass & $<<1 \%$ \\
H(n,n) Reference XS & $<0.5 \%$ \\
Detector Efficiency & \\
${ }^{3} \mathrm{H}(\mathrm{p}, \mathrm{n}) d \sigma / d \Omega$ & $\sim 3 \%$ \\
Attenuation and Multiple Scattering & \\
n $\sigma$ & $0.3 \%$ \\
sample dimensions & $0.3 \%$ \\
sample-cell distance & $0.2 \%$ \\
method (neutrons) & $<5 \%$ \\
method $(\gamma)$ & $<5 \%$ \\
\hline
\end{tabular}
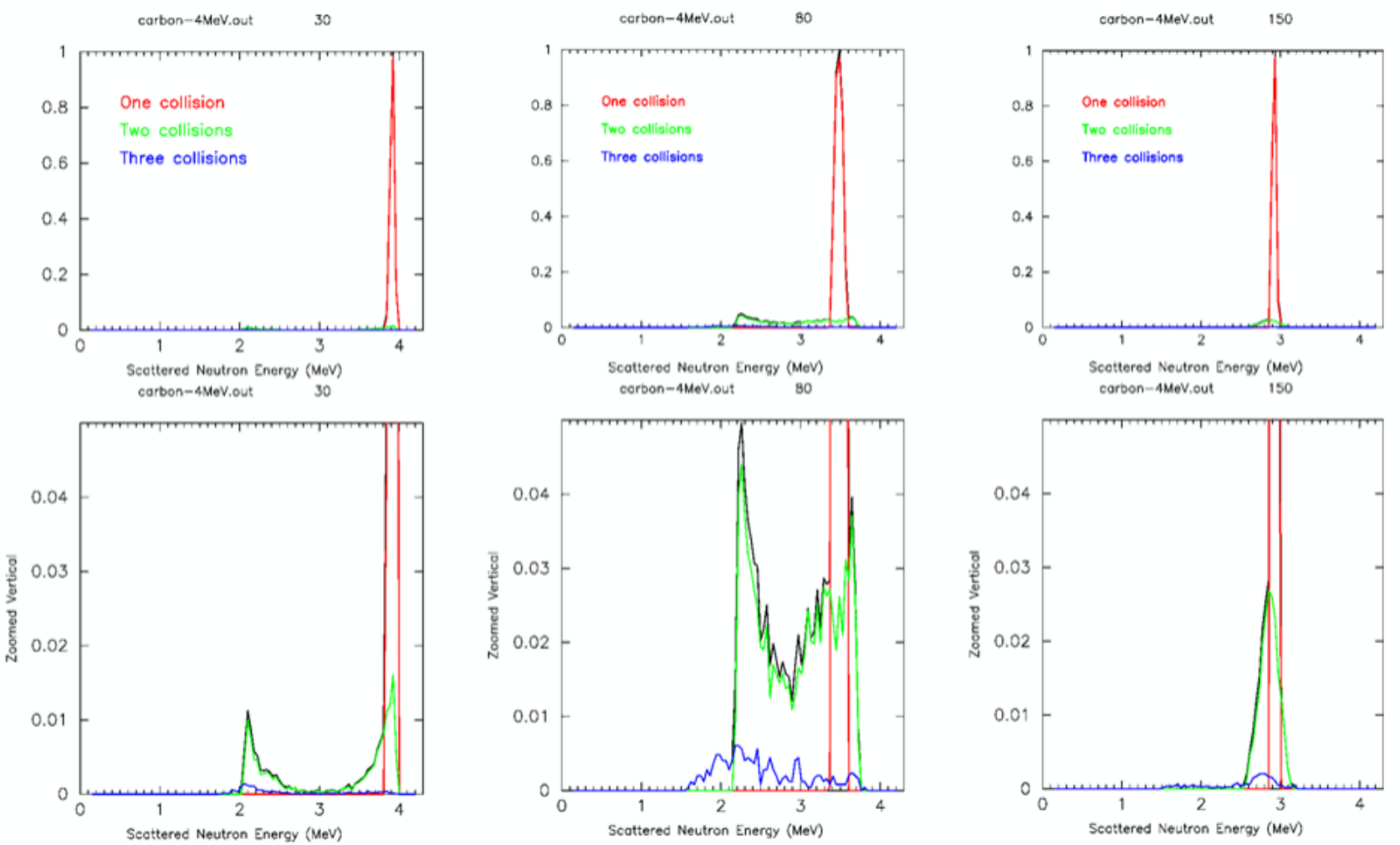

Figure 3. MCNP calculations of TOF spectra of ${ }^{12} \mathrm{C}(\mathrm{n}, \mathrm{n})$ at $\mathrm{E}_{n}=4.0 \mathrm{MeV}$ at angles of $30^{\circ}, 80^{\circ}$, and $150^{\circ}$. Contributions from 1-, 2-, and 3-scatterings in the carbon sample are shown separately in each subfigure. The bottom row of figures is that of the top row with a zoomed vertical scale to make left-side features more visible.

scattering yield could be missed, we calculate the cumulative strength up to a given energy as

$$
\text { cumulative strength }(E)=\int_{0}^{E} \text { yield }(E) d E \text {. }
$$

Results for 4.0 MeV neutrons on ${ }^{12} \mathrm{C}$ are displayed in Fig. 4. Not surprisingly, very little yield would be missed at $150^{\circ}$. Approximately $5 \%$ of the yield is missed at $30^{\circ}$ and $\sim 17 \%$ missed at $80^{\circ}$. In the general situation, the deficits depend strongly upon the elastic differential cross sections at the chosen bombarding energy, the target nucleus atomic mass, and the sample dimensions.

Spectra and laboratory notes from the 1960s and 1970s measurements are not available. We were able to examine a few spectra used for Ref [3] and find that the effects discussed above would not have been detected. This is likely a widespread issue among datasets for low-A targets of that era. The impact upon the differential cross section 

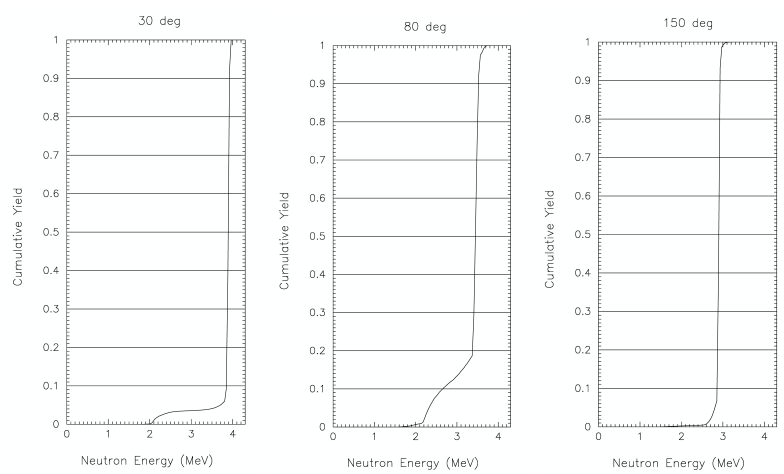

Figure 4. Cumulative strength calculations of the elastic peak in ${ }^{12} \mathrm{C}(\mathrm{n}, \mathrm{n})$ TOF spectra at $\mathrm{E}_{n}=4.0 \mathrm{MeV}$ at angles of $30^{\circ}, 80^{\circ}$, and $150^{\circ}$.

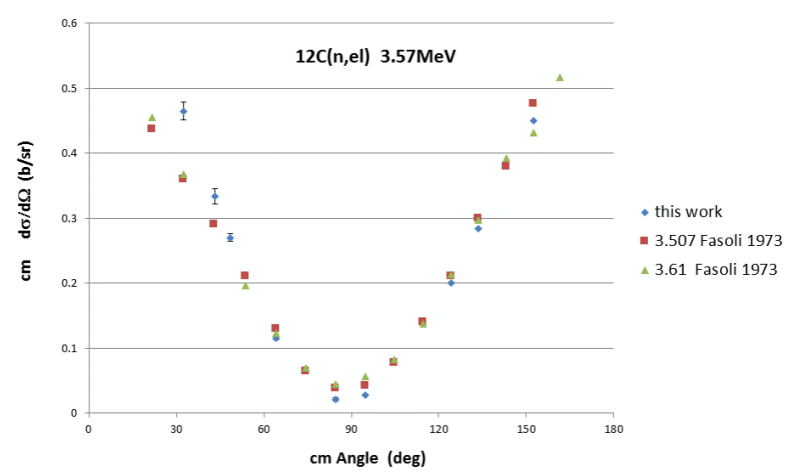

Figure 5. Elastic angular distribution ${ }^{12} \mathrm{C}(\mathrm{n}, \mathrm{n})$ at $3.57 \mathrm{MeV}$. Present results are compared with those of Ref. [5].

is illustrated in Fig. 5 and 6 for two completely different energy regions. The effect is easily noticable at forward angles, but close inspection in the minimum at $\mathrm{E}_{n}=3.57$ $\mathrm{MeV}, \sim 90^{\circ}$ indicates a large effect there also.

The nature of the effect depends upon the target and its cross sections. Simulations on other targets were performed and results summarized in Table 2. The left-side problem is more prominent for low-A targets because of the larger kinematic shift in scattered neutron energy. Note that how one judges the fit to the tailing on the elastic TOF peak impacts cross sections extracted for nearby inelastic peaks.

\section{Summary}

The need for high-quality measured cross sections continues to increase. While uncertainties of 10 - $20 \%$ are easily achievable, further improvement requires careful examination of all analysis methods and procedures. In this manuscript, we have examined the challenge of extracting proper yields from scattered neutron TOF spectra. We find for lighter nuclei, peak shapes arise which are not easily treatable with standard fitting codes. A specialized fitting code which is guided with Monte Carlo predicted response functions seems merited.

\section{Acknowledgements}

Research at the University of Kentucky Accelerator Laboratory is supported by a contract from the U.S. Department of Energy-NEUP, the U.S. National Science Foundation, the USNA James Kinnear Fellowship, and the Cowan Physics Fund at the University of Dallas.

\section{References}

[1] S.F. Hicks, et al., Studies of ${ }^{54,56}$ Fe Neutron Scattering Cross Sections (CGS-15, Dresden, 2014)

[2] X-5 Monte Carlo Team, MCNP, A General Monte Carlo N-Particle Transport Code, Version 6, Tech. Rep. LA-UR-13-22934 (LANL, Apr 2013)

[3] W. Galati, et al., Phys. Rev C 5, 1508 (1972)

[4] M.B. Chadwick, et al., LANL-ORNL ${ }^{12} C$ ENBF/BVII.1 Evaluation(Jun 1976)

[5] U. Fasoli, et al., Nucl. Phys. 205, 302 (1973)

[6] F. Demanins, et al., INFN Reports: Low Energy Nuclear Physics Series 73/2 (1973)

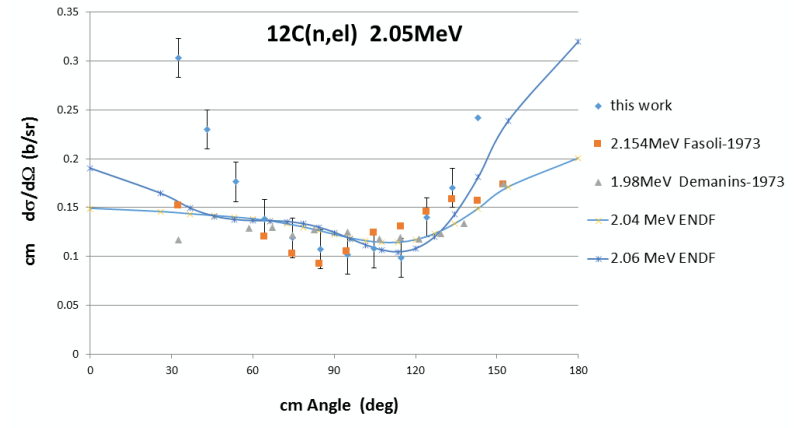

Figure 6. Elastic angular distribution ${ }^{12} \mathrm{C}(\mathrm{n}, \mathrm{n})$ at $2.05 \mathrm{MeV}$. Present results are compared with the ENDF/B-VII.1 compilation [4] and those of Refs. [5, 6]. 